\title{
Circular RNA hsa_circ_0026552 inhibits the proliferation, migration and invasion of trophoblast cells via the miR-331-3p/TGF- $\beta R 1$ axis in pre-eclampsia
}

\author{
LI SHAN $^{1}$ and XIAOFEI HOU ${ }^{2}$ \\ ${ }^{1}$ Department of Obstetrics, Yantaishan Hospital; ${ }^{2}$ Department of Prenatal Screening Laboratory, \\ The Affiliated Yantai Yuhuangding Hospital of Qingdao University, Yantai, Shandong 264000, P.R. China
}

Received January 6, 2021; Accepted May 24, 2021

DOI: $10.3892 / \mathrm{mmr} .2021 .12438$

\begin{abstract}
Globally, pre-eclampsia (PE) is a gestational disorder that causes increased morbidity of the fetus and mortality induced by pregnancy. Despite various studies, the understanding of the causes or mechanism of the development of PE remains elusive. Thus, the present study aimed to investigate the role of circular (circ)RNA hsa_circ_0026552 (hsa_circ_0026552) in the development of PE and its mechanism of regulation. hsa_circ_0026552 differential expression in PE tissue data and clinical samples were analyzed and it was observed that hsa_circ_0026552 is highly upregulated in PE samples. Furthermore, miR-331-3p was detected as an hsa_circ_0026552 target miRNA and TGF- $\beta R 1$ gene as a target of miR-331-3p. These results were confirmed using various assays, including dual-luciferase reporter, reverse transcription-quantitative PCR and RNA pull-down assay. It was observed that miR-331-3p expression was negatively correlated to hsa_circ_0026552 relative expression, while TGF- $\beta$ R1 expression was positively correlated to hsa_circ_0026552 expression evaluated by Pearson's correlation test. The functional experiments, including Cell Counting Kit-8, colony formation and Transwell assay, showed that silencing hsa_circ_0026552 could significantly strengthen the proliferation, migration and invasion of the trophoblastic HTR-8/SVneo cells, but the subsequent overexpression of hsa_circ_0026552 reversed this. Mechanistically, it was concluded that hsa_circ_0026552 acts as a miR-331-3p sponge to upregulate TGF- $\beta \mathrm{R} 1$ expression in trophoblasts and is involved significantly in PE development and progression in
\end{abstract}

Correspondence to: Dr Xiaofei Hou, Department of Prenatal Screening Laboratory, The Affiliated Yantai Yuhuangding Hospital of Qingdao University, 20 Yuhuangding Road, Yantai, Shandong 264000, P.R. China

E-mail: xiao2fei68@163.com

Key words: hsa_circ_0026552, miR-331-3p, pre-eclampsia, TGF- $\beta$ R1, trophoblasts pregnant women. The circRNA hsa_circ_0026552 could be a novel therapeutic target and prognostic biomarker for PE.

\section{Introduction}

PE is a specific gestational disorder characterized by the manifestation of hypertension and proteinuria after 20 weeks of conception $(1,2)$ and is the primary cause of increased fetal morbidity and gestation-induced mortality, affecting $\sim 3$ to $8 \%$ of pregnant women globally (3-5). PE is defined according to the International Society for the Study of Hypertension in Pregnancy as systolic ( $\geq 140 \mathrm{mmHg}$ ) and diastolic $(\geq 90 \mathrm{mmHg})$ blood pressure (6) with proteinuria of $\geq 30 \mathrm{mg} / \mathrm{mol}$ protein or without proteinuria or with austere clinical characteristics after 20 weeks of pregnancy in women that previously had a normal blood pressure (6). Its causes remain mysterious. Dysfunction of the placenta, improper maternal vascular destruction, dysregulation of oxygen and aberrant spiral artery re-modeling are involved in $\mathrm{PE}$ pathogenesis $(7,8)$. Notably, the dysfunction of the placenta is now considered as the potentially main cause of PE (7). However, the fundamental course of placental dysfunction in the development and progression of PE remains to be elucidated (9). Furthermore, either shallow or extensive trophoblastic invasion of extravillous trophoblasts (EVTs) at the maternal-fetal line is known as a primary cause of placentation failure, leading to the occurrence of PE $(10,11)$. It has been demonstrated that the migratory activity of restricted EVT cells in maternal decidua may hinder trophoblast function, thus inducing PE (12). As a result, it is important to clarify the pathophysiological mechanisms in the abnormal migration and invasion of the trophoblast and EVTs $(13,14)$.

Circular (circ)RNAs, a subclass of an endogenous form of non-coding RNA, have conserved closed continuous loop structures (without free $3^{\prime}$ and $5^{\prime}$ ends) and are highly stable and conserved (15-17). CircRNAs constitute a significant part of the mammalian transcriptome and can regulate the expression of specific genes through transcriptional and post-transcriptional modulation and post-translational mechanisms, broadly altering the expression and function of proteins and thus being involved in several cellular mechanisms, for instance, inflammation, apoptosis, cell differentiation, proliferation, migration and invasion (17). 
Emerging evidence has suggested that circRNAs are a significant regulator in several diseases (18), including cancers $(19,20)$ and researchers are now giving attention to their regulatory effect in $\mathrm{PE}$, as they have been reported as an important PE biomarkers (21-23). For instance, Jiang et al (23) showed that has_circ-0001855 and has_circ_0004904 were markedly raised in individuals with $\mathrm{PE}$ and were considered as a possible PE biomarker for subjects by merging with the pregnancy-associated plasma protein A2 (PAPP-A), recognized as homolog of PAPPA in the metzincin superfamily of pappalysins which has attracted considerable attention due to its role in PE pathogenesis. Furthermore, Shen et al (24) described that circTRNC18 inhibits the epithelial-mesenchymal transition (EMT) and migration of trophoblast cells by regulating miR-762-mediated expression of Grh12 in PE. Hsa_circ_0026552 (accession number: NM_002178) is located at chr12: 53494495-53496128 and was found by Salzman et al (25) in 2013 in lung cancer cells, human fibroblasts, human normal epidermal keratinocytes and other types of cells with abnormal expressions, suggesting that it may serve a role in regulating cell phenotype. However, no study has reported on the role of hsa_circ_0026552 in PE. Hence, this present study was primarily to determine the role of hsa_circ_0026552 in the progression of PE.

micro (mi)RNAs are involved in the regulation of mRNA expression by targeting them (26). Therefore, they have attracted widespread attention and become a new type of biomarker for the treatment of PE through the mRNA-miRNA-mRNA axis. For example, long non-coding (lnc)RNA MALAT1 regulates trophoblast cell migration and invasion via the miR-206/IGF-1 axis (27), decreased lncRNA ZEB2-AS1 in pre-eclampsia controls the invasive and migratory abilities of the trophoblastic cell line HTR-8/SVneo via the miR-149/PGF axis (28). miR-331-3p serves an important role in inhibiting various disease progressions and is a critical biomarker. For instance, miR-331-3p suppresses cell proliferation in triple-negative breast cancer cells by downregulating neuropilin 2 (NRP2) (29) and suppresses cell invasion and migration in colorectal carcinoma (30). In addition, miR-331-3p inhibits the hepatocellular carcinoma Bel-7402 cell line by downregulation of E2F Transcription Factor 1 (31). However, there are no studies on miR-331-3p in preeclampsia, to the best of the authors' knowledge.

TGF- $\beta$ s are members of a large superfamily of cytokines, including activins, inhibitors and bone morphogenic proteins (32). In addition, the TGF- $\beta$ is a multifunctional cytokine that serves an essential role in the pathogenesis of PE (33). For example, miRNAs serve as common regulators of the TGF- $\beta$ pathway in the preeclamptic placenta and cadmium-treated trophoblasts: Links between the environment, the epigenome and PE (34). Binding of TGF- $\beta$ to TGF- $\beta$ receptor which consists of the subunits TGF- $\beta$ R 1 , a key receptor of signaling pathway, is a target gene of miR-331-3p (35). Previous studies have shown that TGF- $\beta$ R1 serves a role in the gene signal axis in a number of diseases, including PE, and can serve as the downstream target gene of multiple mRNAs $(36,37)$. For instance, Kim et al (33) indicated that the TGF- $\beta \mathrm{R} 1$ polymorphism may be a genetic risk factor for PE and IUGR-complicated PE. In addition, the serum levels of TGF- $\beta$ R1 may contribute to the etiopathology of PE (38). Hence the aim of the present study to understand the deep molecular mechanism of PE.

The present study aimed to explore the role and the potential regulatory mechanism of hsa_circ_0026552 in PE progression. The results demonstrated that hsa_circ_0026552 suppressed cell proliferation and metastasis and invasion by regulating miR-331-3p/TGF- $\beta \mathrm{R} 1$ axis, a critical insight to identify novel molecular targets for the treatment of patients with PE.

\section{Materials and methods}

Analysis of pre-eclampsia dataset from gene expression omnibus (GEO) and clinical sample collection. Pre-eclampsia datasets with the accession number GSE96985 were retrieved from the GEO website and analyzed for differential expression using the heatmap method (39) by R project software (r-project.org/; version: 4.0). Placenta tissues obtained from pre-eclampsia whose age distribution was $28.4 \pm 3.3(n=30)$ and normal pregnant women whose maternal age at delivery is $27.6 \pm 2.9(n=30)$ at the Yantaishan Hospital between June 2015 and November 2019 were washed in PBS and kept at $-80^{\circ} \mathrm{C}$ for further experimentation. The diagnosis criteria for severe PE were as follows: Systolic pressure $\geq 160 \mathrm{mmHg}$ and/or diastolic pressure $\geq 110 \mathrm{mmHg}$ on at least two occasions with $4 \mathrm{~h}$ apart, accompanied by severe proteinuria ( $>5 \mathrm{~g} / 24 \mathrm{~h}$ urine specimen or $3+$ on $\geq 2$ random samples collected $4 \mathrm{~h}$ apart). All the participants had no vaginal delivery, chronic nephritis, diabetes, heart and autoimmune diseases or cancer. The present study was authorized by the Ethics Committee of Yantaishan Hospital (approval no. KY-E-2020-1-10) and informed written consent was provided by all participants as per the guidelines of the Declaration of Helsinki.

Microarray analysis. Microarray datasets GSE96985 which includes four normal tissues and three PE tissues at platform GPL19978 were used to identify differentially expressed circRNAs through $\mathrm{R}$ software of limma package (R-project. org/; version 4.0) (40) with adjusting $\mathrm{P}<0.05$ and Ifold changel $>2$. Cluster analysis was performed by using the pHeatmap package in R software and Z-scores (r-project.org/; version: 4.0) was used for normalization. The top 20 most upregulated or downregulated genes were shown in the heatmap and the final value displayed as the units processed by Z-scores.

Cell lines and transfection. The human trophoblast cell line HTR-8/SVneo was acquired from the American Type Culture Collection and cultured in DMEM-F12 medium (HyClone; Cytiva), supplemented with FBS (10\%) and penicillin/streptomycin (1\%; Gibco; Thermo Fisher Scientific, Inc.) in a humidified incubator (at $37^{\circ} \mathrm{C}$ and $5 \% \mathrm{CO}_{2}$ ) and then sub-cultured (ratio of $1: 3$ ) at $80-90 \%$ confluence to generate a new culture with a lower density of HTR-8/SVneo cells. For cell transfection, hsa-circ-002655 was inserted into an overexpression plasmid pcDNA 3.1(+) (OE-hsa-circ-002655, Sigma-Aldrich; Merck KGaA) and an empty vector [pcDNA3.1(+)]. Circ-002655 and TGF- $\beta$ R1 knock-down (si-00265521, si-TGF- $\beta$ R1) procedure was performed by using validated Stealth RNAi siRNA (Invitrogen; Thermo Fisher Scientific, Inc.) according to the manufacturer's instructions. 
miR-331-3p inhibitor and miR-331-3p mimics, as well as their $\mathrm{NC}$ negative controls ( $\mathrm{NC}$; mimics-NC, inhibitor-NC) were separately designed by Shanghai GeneChem Co., Ltd. Briefly, $4 \times 10^{5} \mathrm{HTR} 8 / \mathrm{SVneo}$ cells were seeded in a serum medium on the day of operation and $20 \mathrm{nM}$ siRNA and miRNA transfection solution was mixed with Lipofectamine ${ }^{\mathrm{TM}} 2000$ (Invitrogen; Thermo Fisher Scientific, Inc.) and serum-free media according to the manufacturer's protocol to produce a final volume of $100 \mu \mathrm{l}$ transfection reagent mixture at room temperature for $15 \mathrm{~min}$. After $48 \mathrm{~h}$ of transfection, the medium was replaced and cells were harvested and at $72 \mathrm{~h}$ post-transfection, the transfection efficiency was evaluated before further experiments (39). The sequences were: hsa_circ_0026552, si-\#1: 5'-GTCGCTGTTGCAGAGGAGAAT-3'; si-\#2: 5'-GGT CGCTGTTGCAGAGGAGAA-3'; si-NC, 5'-CAACAAGAT GAAGAGCACCAA-3'; mir-331-3p mimics, 5'-GCCCCU GGGCCUAUCCUAGAA-3'; mimics-NC, 5'-CAGUACUUU UGUGUAGUACAA-3'; miR-331-3p inhibitor, 5'-GUCAUG AAAACACAUCAUGUU-3'; inhibitor-NC, 5'-CGAACG UGUCACGUTT-3'; si-TGF- $\beta R 1$ 1, 5'-AAAAUUGUCUUU UGUACAGAG-3'; si-NC, 5'-CAACAAGATGAAGAGCAC CAA-3'.

Isolation of total RNA and reverse transcription quantitative $(R T-q)$ PCR. Total RNA from HTR-8/SVneo cells and the placental tissues was isolated with TRIzol (Thermo Fisher Scientific, Inc.). cDNA synthesis was performed with the Reverse Transcription kit (Takara Biotechnology Co., Ltd.) according to the manufacturer's protocols. and detection of mRNA with the SYBR-Green PCR kit (Takara Biotechnology Co., Ltd.) in accordance with the manufacturer's protocols. qPCR was used with an ABI7900 system (Applied Biosystems; Thermo Fisher Scientific, Inc.). The PCR amplification involved denaturation at $94^{\circ} \mathrm{C}$ for $1 \mathrm{~min}, 40$ cycles of $95^{\circ} \mathrm{C}$ for $30 \mathrm{sec}$ and $60^{\circ} \mathrm{C}$ for $1 \mathrm{~min}$. GAPDH was used as the internal control for has-circ-0026552 and TGF- $\beta$ R 1, U6 was used as the internal control miR-331-3p (41). The data were processed using a $2^{-\Delta \Delta \mathrm{Cq}}$ method (42). The primer sequences were as follows: circ_0026552 forward, 5'-CGCTGGTTG GAAAGAGTGTT-3' and reverse, 5'-GTCTCTGCGGTTCAC ATAAT-3'; mR-331-3p forward, 5'-TAGCCCCTGGGCCTA TCCTAGAGAACT-3' and reverse, 5'-TCAACTGGTGTC GTGGAGTCGGC-3'; TGF- $\beta$ R1 forward, 5'-TCCAACTAC TGGTTTACCATTGC-3' and reverse, 5'-ACAGCAACTTCT TCTCCCCG-3'; GAPDH forward, 5'-TGCACCACCAAC TGCTTAGC-3' and reverse, 5'-GGCATGCACTGTGGTCAT GAG-3' and U6 forward, 5'-CTCGCTTCGGCAGCACA-3' and reverse, 5'-AACGCTTCACGAATTTGCGT-3'.

Target gene prediction. StarBase v3.0 (http://starbase.sysu. edu.cn/) was used to predict the possible target miRNA of has-circ-0026552 in pre-eclampsia and also the target mRNA.

Cell proliferation assay. The proliferation of HTR-8/SVneo cells was evaluated using CCK-8 assay in accordance with the manufacturer's guidance. Briefly, after $96 \mathrm{~h}$ of transfection, CCK-8 reagent (100 $\mu \mathrm{l}$; Beyotime Institute of Biotechnology) was added into each well. Incubation was then performed for $2 \mathrm{~h}$ at room temperature and the light absorbance was measured at $450 \mathrm{~nm}$ using a microplate reader (BioTek Instruments,
Inc.). Each group was replicated five times and the assay was performed three times independently.

Colony formation assay. Cells were seeded ( $5 \times 10^{3} /$ well) in a six-well plate and transfected with pcDNA3.1-has-circ-0026552 for $96 \mathrm{~h}$. After two weeks, the cells were washed with PBS, fixed with ethanol for $30 \mathrm{~min}$ at room temperature and finally stained with $1 \%$ crystal violet for $20 \mathrm{~min}$ at room temperature (Sigma-Aldrich; Merck KGaA) for counting colony numbers (diameter $\geq 100 \mu \mathrm{m}$ ). The number of colonies formed was analyzed using a light microscope (magnification, x50; Olympus Corporation). The assay was performed three times independently.

Cell migration and invasion assay. To determine the migration and invasion of the trophoblastic cell lines, the cells were treated or transfected with different oligonucleotides and then suspended, without FBS, in RPMI-1640 medium. Afterward, the cells were seeded in the upper compartment of the Transwell inserts ( $8 \mu \mathrm{m}$-pore size; Sigma-Aldrich; Merck KGaA) coated with or without Matrigel for invasion and migration assay at room temperature for $30 \mathrm{~min}$, respectively, while the lower chamber was occupied with a complete medium. After incubation for $96 \mathrm{~h}$ at $37^{\circ} \mathrm{C}$, non-migratory/non-invasive cells attached to the upper portion of the inserts were removed using cotton swabs, while the migrated or invaded cells were fixed with $4 \%$ paraformaldehyde (Thermo Fisher Scientific, Inc.) at room temperature and then stained with crystal violet $(0.5 \%)$ for $10 \mathrm{~min}$ at room temperature. Finally, under a light microscope (Leica DMIL-PH1; Leica Microsystems GmbH), the invaded/migrated cells were counted in five random fields of each filter membrane.

Dual-luciferase reporter assay. The has_circ_0026552 sequence and TGF- $\beta$ R 1 3'UTR sequence including miR-331-3p-binding sites was cloned into a pGL3 vector (Promega Corporation) creating a pGL3-has_circ_0026552 wild type (WT) plasmid (has-circ-0026552-WT) and pGL3-TGF- $\beta$ R1 WT plasmid (TGF- $\beta$ R1-WT), respectively. miR-331-2p binding sites were mutated in both has_circ_0026552 and TGF- $\beta$ R1 3' UTR sequence and then sub-cloned into the pGL3 vector to generate their mutant type (MUT) vector. To conduct the luciferase reporter assay, the HTR-8/SVneo cells were co-transfected with miR-331-3p mimics or miR-NC and the pGL3-has_circ_0026552 and pGL3-TGF- $\beta$ R1 reporter construct using Lipofectamine ${ }^{\mathrm{TM}}$ 2000 (Invitrogen; Thermo Fisher Scientific, Inc.) at $37^{\circ} \mathrm{C}$. After transfection (96 h), the relative luciferase activity of the cells was evaluated with the Dual-Luciferase Reporter Assay System (Promega Corporation) and subsequently normalized to the Renilla luciferase reporter activity.

Western blot analysis. Radioimmunoprecipitation assay (RIPA) lysis buffer (Beyotime Institute of Biotechnology) was used to extract proteins and the concentrations were determined with Bicinchoninic Acid (BCA) assay (Bio-Rad Laboratories, Inc.). Equivalent amounts of proteins (40 $\mu \mathrm{g})$ were separated by $10 \%$ SDS-PAGE electrophoresis and later moved onto polyvinylidene fluoride (PVDF) membranes. The PVDF membranes were blocked at room temperature 

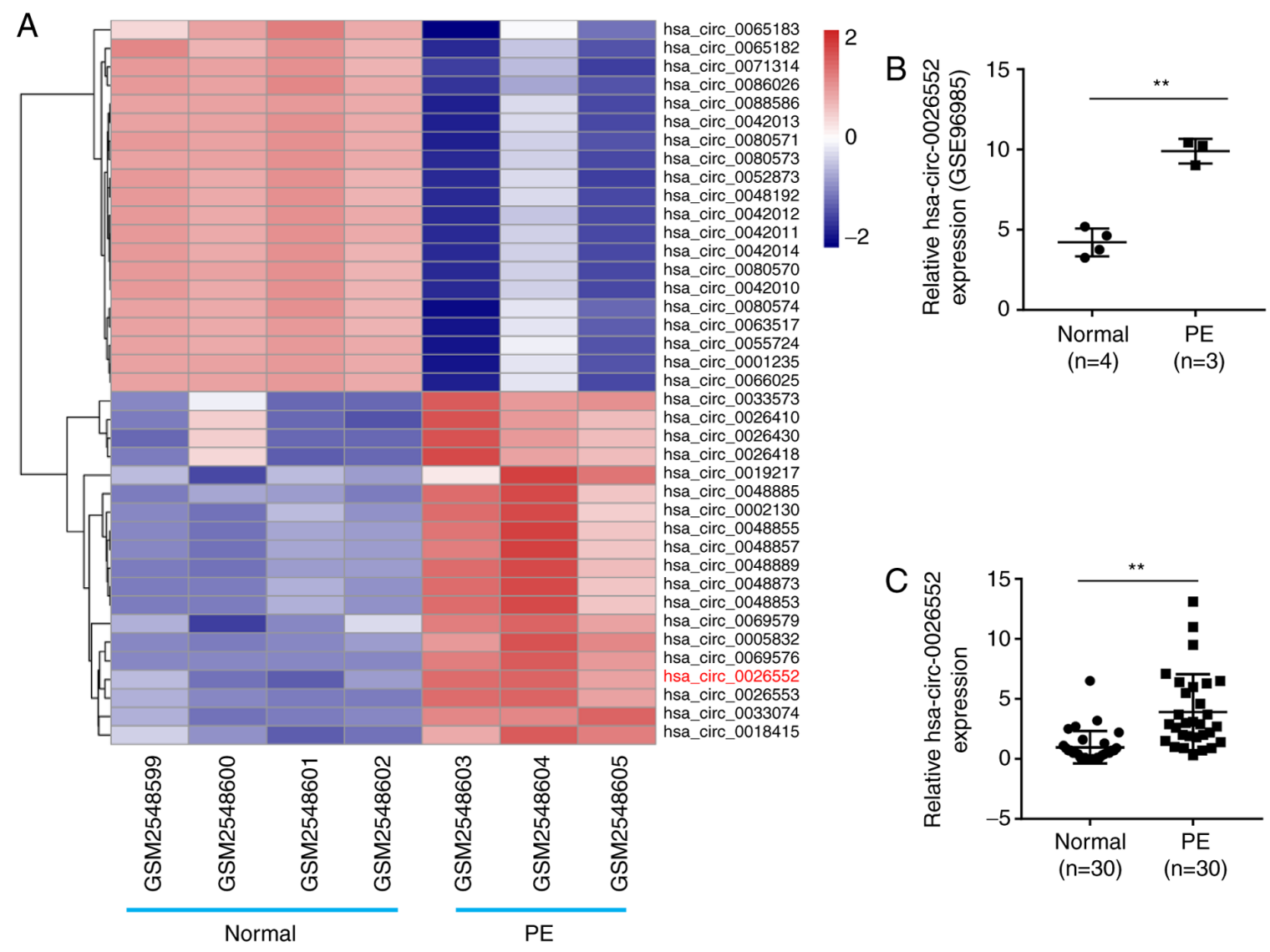

Figure 1. circRNA hsa_circ_0026552 expression in PE placental tissue samples. (A) Heatmap analysis of hsa_circ_0026552 expression in PE tissue samples downloaded from the Gene Expression Omnibus database and normal healthy control tissue samples. (B) circRNA hsa_circ_0026552 was among the top 20 most differentially expressed circRNA in the three PE tissue samples as compared with the normal ones was detected by RT-qPCR. (C) RT-qPCR analysis of hsa_circ_0026552 expression in 30 PE clinical samples. The hsa_circ_0026552 was significantly upregulated in PE samples compared with the normal ones. ${ }^{* *} \mathrm{P}<0.01$. circRNA, circular RNA; PE, pre-eclampsia; RT-qPCR, reverse transcription quantitative PCR.

with $5 \%$ skimmed milk for $1 \mathrm{~h}$. Incubation was performed overnight using appropriated primary antibodies (rabbit polyclonal antibodies for TGF- $\beta$ R1 cat. no. 41896S; 1:1,000; Cell Signaling Technology, Inc.) and $\beta$-actin (cat. no. ab5694; 1:2,000; Abcam) at $4^{\circ} \mathrm{C}$. Afterward, the PVDF membranes in horseradish peroxidase-conjugated secondary antibodies (cat. no. ab6721, 1:2,000; Abcam) was incubated for $2 \mathrm{~h}$ at room temperature. Protein blots were visualized with the ECL-Plus Western Blot Analysis Detection System (Thermo Fisher Scientific, Inc.) and the band densities were quantified using ImageJ software (V1.8.0.112; National Institutes of Health).

Biotinylated RNA pull-down assay. Biotinylation of miR-NC and miR-331-3p to make Bio-miR-NC and Bio-miR-331-3p was performed by Shanghai GenePharma Co., Ltd.. Subsequently, HTR8/SVneo cells were transfected with biotinylated oligonucleotides. Next, the treated HTR8/SVneo cells were lysed by lysis buffer (Thermo Fisher Scientific, Inc.), incubated with streptavidin-coated magnetic beads (Invitrogen; Thermo Fisher Scientific, Inc.) according to the manufacturer's protocol and eluted after $96 \mathrm{~h}$. Finally, the eluted biotin-coupled RNA complex was subsequently washed with wash buffer (Thermo Fisher Scientific, Inc.), collected by centrifugation at $11,100 \mathrm{x}$ g for $10 \mathrm{~min}$ at room temperature and assessed by RT-qPCR.
Statistical analysis. SPSS version 20.0 (IBM Corp.) or GraphPad Prism 6 (GraphPad Software, Inc.) was employed for the statistical analysis and all experimental data were presented as mean \pm standard deviation. Unpaired t-test was used to identify the significant difference between two groups and the significance among multiple groups was calculated by one way ANOVA with Tukey's correction. Pearson's correlation test was used to evaluate the correlations between different RNA expressions level in PE clinical samples. All the experiments were repeated three times and all data in each histogram were normalized to the mean value of the control group and were presented as fold change, unless otherwise indicated. $\mathrm{P}<0.05$ was considered to indicate a statistically significant difference.

\section{Results}

Hsa_circ_0026552 is significantly upregulated in the placental tissues of pre-eclampsia pregnant women. At the beginning of the present study, the pre-eclampsia dataset with the accession number GSE96985 was downloaded from the Gene Expression Omnibus (GEO) website (ncbi.nlm.nih. gov/geo/) and analyzed for differential expression using the heatmap method. As shown in Fig. 1A, hsa_circ_0026552 (in red) was among the top 20 most differentially expressed circRNA in the three PE tissue samples compared with that 

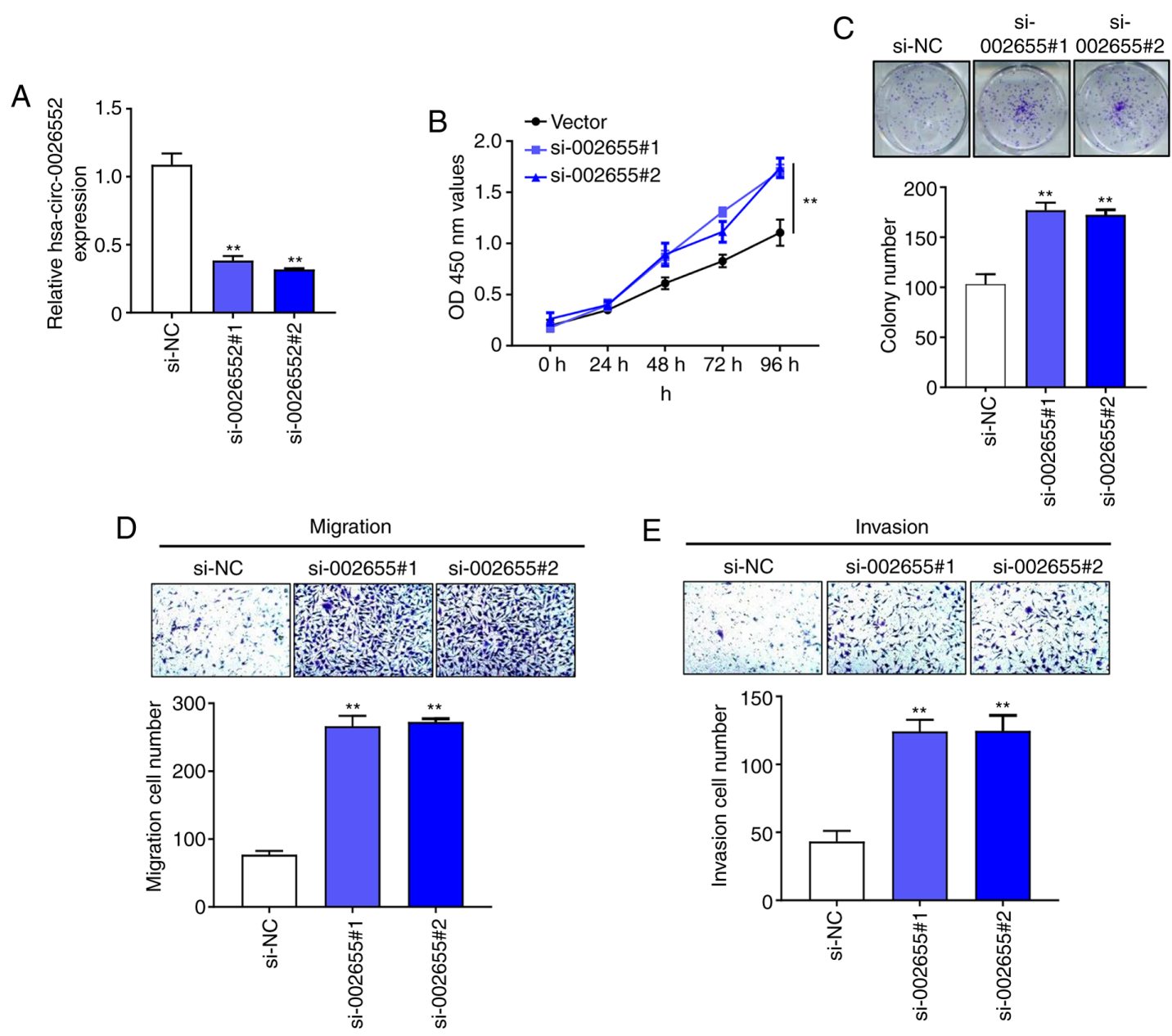

Figure 2. circRNA hsa_circ_0026552 knockdown inhibits proliferation, migration and invasion of trophoblasts. (A) Reverse transcription quantitative PCR showed the hsa_circ_0026552 expression in HTR-8/SVneo cells following knockdown with si-0026552. (B) The proliferative ability of HTR-8/SVneo cell following hsa_circ_0026552 knockdown was evaluated using CCK-8 assay. (C) Colony formation assay was used to analysis proliferative ability of HTR-8/SVneo cells following hsa_circ_0026552 knockdown. Transwell assay for detecting the (D) migration and (E) invasion ability of trophoblasts following hsa_circ_0026552 knockdown. Magnification, x200. All experimental data are shown as mean \pm standard deviation of three different experiments ${ }^{* *} \mathrm{P}<0.01$. circRNA, circular RNA; PE, pre-eclampsia; si, short interfering.

in the normal tissue samples. Further expression analysis of the hsa_circ_0026552 in the GSE96985 dataset showed that hsa_circ_0026552 is significantly upregulated in PE tissues in comparison to that in the normal tissue samples $(\mathrm{P}<0.01$; Fig. 1B). The expression level of hsa_circ_0026552 was subsequently detected in $30 \mathrm{PE}$ tissues collected from PE pregnant women using the RT-qPCR. It was found that hsa_circ_0026552 was significantly overexpressed in PE tissue samples compared with that in the normal tissue samples collected from pregnant women $(\mathrm{P}<0.01$; Fig. $1 \mathrm{C})$. These data suggested that the circRNA hsa_circ_0026552 might serve a role in the development of $\mathrm{PE}$ in pregnant women.

Knockdown of hsa_circ_0026552 suppresses the proliferation, migration and invasion of trophoblasts. To understand the biological function of hsa_circ_0026552 in the development of PE, hsa_circ_0026552 was knocked down in HTR-8/SVneo cells. RT-qPCR and western blot were used to verify whether GAPDH can serve as an internal control (Fig. S1A and B). The results showed that there was no significant difference in the expression of GAPDH under different conditions, indicating that it could be used as an experimental internal control.
RT-qPCR analysis showed that hsa_circ_0026552 knockdown (with si-0026552) markedly reduced hsa_circ_0026552 expression in the cells $(\mathrm{P}<0.01$; Fig. 2A). Furthermore, CCK-8 and colony formation assays revealed that silencing hsa_circ_0026552 significantly increased the proliferation of the HTR-8/SVneo cells in a time-dependent manner and the number of colonies formed in the cells $(\mathrm{P}<0.001$; Fig. 2B and C). Transwell assay was performed to understand the function of the hsa_circ_0026552 in the migration and invasion of HTR-8/SVneo cells. The results indicated that hsa_circ_0026552 knockdown significantly increased the migration and invasion ability of the HTR-8/SVneo cells ( $\mathrm{P}<0.001$; Fig. 2D and E).

Overexpression of hsa_circ_0026552 inhibits the proliferation, migration and invasion of trophoblasts. The biological effect of the overexpression of hsa_circ_0026552 on cell proliferation, migration and invasion was also determined. To achieve this, pcDNA3.1-hsa_circ_0026552 was transfected to the HTR-8/SVneo cells. As shown in Fig. 3A, the relative expression of hsa_circ_0026552 was significantly upregulated in the HTR-8/SVneo cells transfected with hsa_circ_0026552 


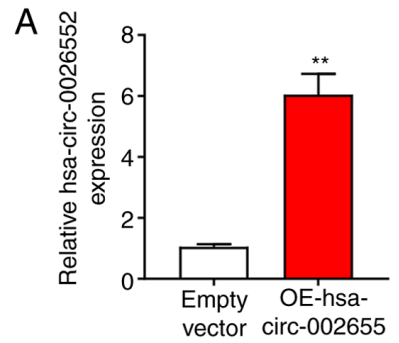

$\mathrm{D}$
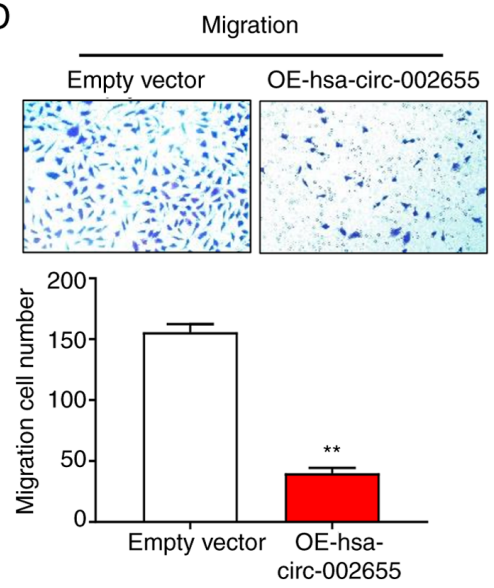

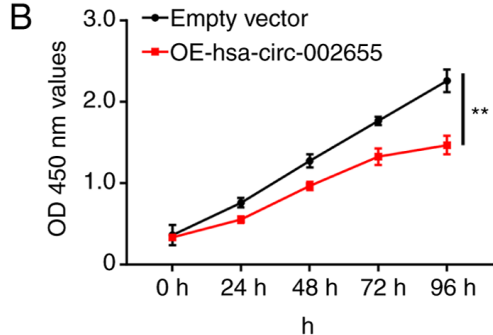

C
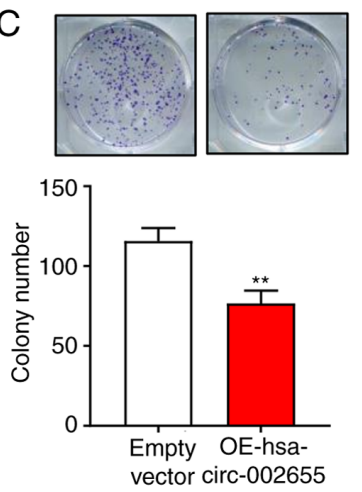

E
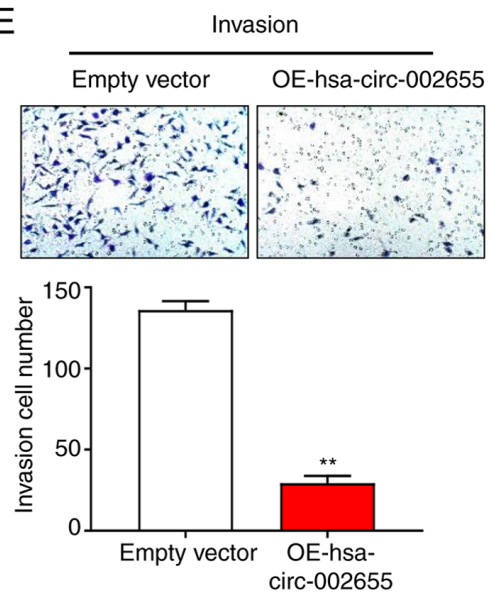

Figure 3. Overexpression of circRNA hsa_circ_0026552 inhibits the proliferation, migration and invasion of trophoblasts. (A) Reverse transcription quantitative PCR detection of hsa_circ_0026552 expression following its overexpression in HTR-8/SVneo cells. (B) CCK-8 and (C) colony formation assays were used to detect HTR-8/Svneo cell proliferation following transfection with hsa_circ_0026552 overexpression plasmid. Detection of (D) migration and (E) invasion ability of the HTR-8/Svneo cells, following transfection with hsa_circ_0026552 overexpression plasmid, using Transwell assay. Magnification, x200. All experimental data are shown as mean \pm standard deviation of three different experiments ${ }^{* *} \mathrm{P}<0.01$. circRNA, circular RNA; OE, overexpression.

overexpression plasmid (OE-hsa_circ_0026552) compared with those transfected with an empty vector $(\mathrm{P}<0.01)$. CCK-8 and colony formation assays showed that the overexpression of hsa_circ_0026552 significantly reduced the proliferative ability of the HTR-8/SVneo cells and also reduced the number of colonies formed in the cells $(\mathrm{P}<0.01$; Fig. 3B and C). Additionally, it was found that overexpressing hsa_circ_0026552 in the HTR-8/SVneo cells significantly reduced the migration and invasion ability of the cells $(\mathrm{P}<0.01$; Fig. 3D and E).

miR-331-3p is a target of hsa_circ_0026552 in trophoblasts. To better understand the hsa_circ_0026552 mechanism of regulation in PE, it was predicted that hsa_circ_0026552 targeted miRNA using the circinteractome and found that hsa_circ_0026552 harbors a binding site for the miR-331-3p seed region (Fig. 4A) and a number of other targeted miRNAs given in Table I, including miR-1184, miR-1225-3p, miR-1233, miR-1277, miR-1307, miR-145 and miR-1825. A dual-luciferase reporter assay and a biotinylated RNA-pull down assay was performed to confirm this result. As shown in Fig. 4B, the overexpression of miR-331-3p significantly inhibited the luciferase activity of cells co-transfected with the WT pGL3-hsa_circ_0026552 plasmid compared with miR-NC. While the mutation of the miR-331-3p binding site on pGL3-hsa_circ_0026552 (i.e., hsa_circ_0026552 MUT) blocked the inhibiting effect of miR-331-3p on the cells
(P<0.01; Fig. 4B). The result of biotinylated RNA-pull down assay showed that Bio-miR-331-3p significantly pulled down more hsa_circ_0026552 in the HTR-8/SVneo cells than the Bio-NC ( $\mathrm{P}<0.001$; Fig. 4C). Notably, the overexpression of hsa_circ_0026552 significantly inhibited miR-331-3p relative expression $(\mathrm{P}<0.001$; Fig. 4D). In addition, miR-331-3p was found to be significantly downregulated in the PE dataset (GEO number GSE96983) and clinical tissue samples when compared with the normal control samples and its expression was negatively correlated to the hsa_circ_0026552 relative expression level ( $\mathrm{*}<0.05$; Fig. $4 \mathrm{E}$; ${ }^{* *} \mathrm{P}<0.01$; Fig. $4 \mathrm{~F}$; and $\mathrm{P}<0.0001$; Fig. $4 \mathrm{G}$ ). The expression of miR-331-3p in HTR-8/SVneo cells transfected with miR-331-3p mimics and miR-331-3p inhibitor (Fig. S2A and B) was then detected using RT-qPCR. The results revealed that in cells transfected with miR-331-3p mimics, the level of miR-331-3p significantly increased but greatly decreased in cells transfected with miR-331-3p inhibitor. It indicated that the transfection was a success.

Hsa_circ_0026552 regulates TGF- $\beta R 1$ expression in trophoblasts via sponging miR-331-3p. The miR-331-3p target mRNA was also predicted using StarBase and the result showed that miR-331-3p directly targets the 3'UTR region of TGF- $\beta$ R1 (Fig. 5A). Luciferase reporter assay showed that miR-331-3p could significantly inhibit the luciferase activity of HTR-8/Svneo cells co-transfected with the pGL3-TGF- $\beta$ R1 
A

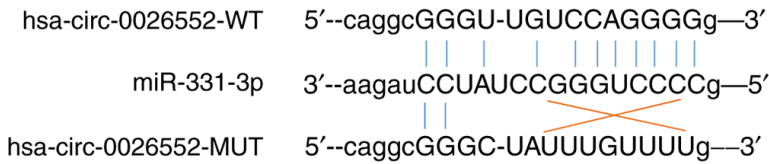

B

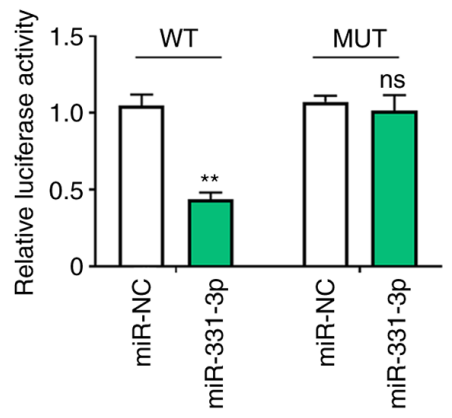

E

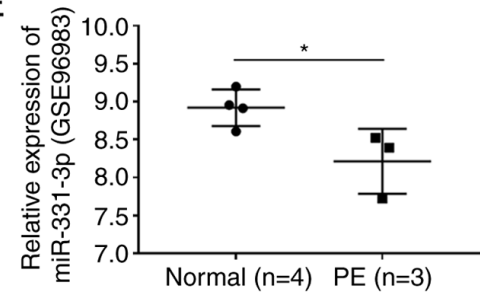

$\mathrm{F}$

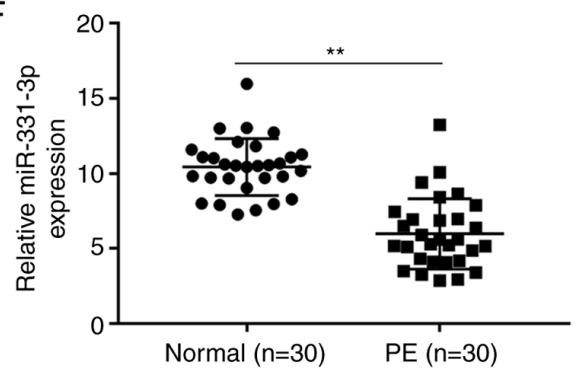

$\mathrm{G}$

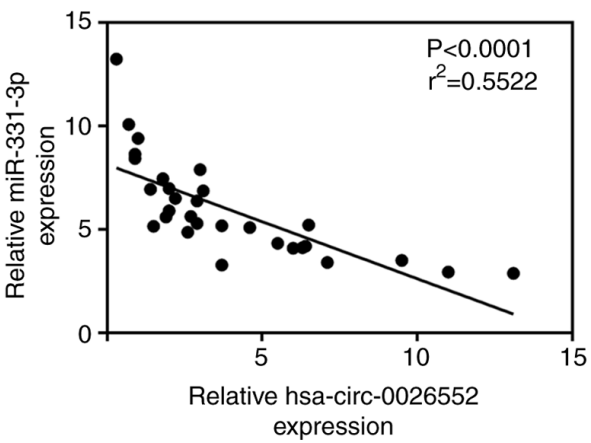

Figure 4. miR-331-3p was a target of hsa_circ_0026552 in trophoblasts. (A) Bioinformatics prediction of hsa_circ_0026552 target miRNA using StarBase database. (B) Dual-luciferase reporter assay for the detection hsa_circ_0026552-miR-331-3p binding ability. (C) Biotinylated RNA pull-down assay was used to conform the interaction between hsa_circ_0026552 and miR-331-3p in the HTR-8/SVneo cells. (D) RT-qPCR detection of miR-331-3p expression after transfecting cells with overexpressed hsa_circ_0026552. (E) Differential expression analysis of miR-331-3p in PE dataset (Gene Expression Omnibus number GSE96983). (F) RT-qPCR detection of miR-331-3p expression in PE clinical tissue samples. (G) Correlation analysis between hsa_circ_0026552 and miR-331-3p expression in PE tissues. The experimental data are shown as the mean \pm standard deviation of at least three independent experiments. ${ }^{*} \mathrm{P}<0.05$; ${ }^{* *} \mathrm{P}<0.01$; ns, non-significant. miR, microRNA; Bio, biotinylated; RT-qPCR, reverse transcription quantitative PCR; PE, pre-eclampsia; WT, wild type; MUT, mutant; OE, overexpression.

WT plasmid, but it had no significant inhibiting effect on that of the cells co-transfected with the pGL3-TGF- $\beta$ R1 MUT plasmid $(\mathrm{P}<0.01$; Fig. 5B). RT-qPCR and western blot analysis showed that inhibiting miR-331-3p expression in the HTR-8/Svneo cell significantly upregulated TGF- $\beta$ R1 relative expression while overexpressing miR-331-3p significantly downregulated TGF- $\beta$ R 1 mRNA and protein expression $(\mathrm{P}<0.01$; Fig. 5C). Similarly, overexpressing hsa_circ_0026552 in the HTR-8/Svneo cell significantly upregulated TGF- $\beta$ R1 mRNA and protein expression which was later downregulated after co-transfecting the hsa_circ_0026552-overexpressed cell with miR-331-3p mimics $(\mathrm{P}<0.01$; Fig. 5D). It was found that TGF- $\beta$ R1 was significantly overexpressed in PE tissue samples relative to the normal healthy tissue samples and its expression was positively correlated to hsa_circ_0026552 relative expression, suggesting that hsa_circ_0026552 could upregulate TGF- $\beta \mathrm{R} 1$ expression in trophoblasts by sponging miR-331-3p ( $\mathrm{P}<0.01$; Fig. 5E and $\mathrm{P}<0.0001$; Fig. 5F $)$. hsa_circ_0026552 regulates the proliferation, migration and invasion of trophoblasts by targeting the miR-331-3p/ TGF- $\beta R 1$ axis. To further confirm the hsa_circ_0026552 mechanism of regulation in PE, the HTR-8/Svneo cells were overexpressed with hsa_circ_0026552 and later co-transfected with miR-331-3p mimics or si-TGF- $\beta$ R1. The results of CCK-8 and colony formation assays showed that the overexpression of hsa_circ_0026552 significantly reduced the proliferation and number of colonies formed in the HTR-8/Svneo cell lines. Co-transfecting the cell with either miR-331-3p mimics or si-TGF- $\beta$ R 1 , however, restored the proliferative ability of the HTR-8/Svneo cell ( $\mathrm{P}<0.01$; Fig. 6A and $\mathrm{B})$. The migration and invasion ability of the HTR-8/Svneo cells were also significantly reduced after overexpressing hsa_circ_0026552, while co-transfecting the cell with miR-331-3p mimics or silencing TGF- $\beta$ R1 in the hsa_circ_0026552-overexpressed cell restored the migration and invasion ability of the HTR-8/Svneo cells $(\mathrm{P}<0.01$; Fig. $6 \mathrm{C}$ and $\mathrm{D})$. These data indicated that 
Table I. Hsa_circ_0026552 targeting miRNAs.

\begin{tabular}{ll}
\hline CircRNA & Target miRNA \\
\hline hsa_circ_0026552 & hsa-miR-1184 \\
& hsa-miR-1225-3p \\
& hsa-miR-1233 \\
& hsa-miR-1277 \\
& hsa-miR-1307 \\
& hsa-miR-145 \\
& hsa-miR-1825 \\
& hsa-miR-198 \\
& hsa-miR-296-5p \\
& hsa-miR-331-3p \\
& hsa-miR-361-3p \\
& hsa-miR-431 \\
& hsa-miR-432 \\
& hsa-miR-486-3p \\
& hsa-miR-515-3p \\
& hsa-miR-515-3p \\
& hsa-miR-519e \\
& hsa-miR-516b \\
& hsa-miR-593 \\
& hsa-miR-622 \\
& hsa-miR-629 \\
& hsa-miR-634 \\
& hsa-miR-651 \\
& hsa-miR-653 \\
& hsa-miR-661 \\
& hsa-miR-663b \\
& hsa-miR-934 \\
\hline & \\
& \\
&
\end{tabular}

circRNA, circular RNA; miR, microRNA.

hsa_circ_0026552 promoted the proliferation, migration and invasion of trophoblastic cell line HTR-8/SVneo by regulating the miR-331-3p/ TGF- $\beta$ R 1 axis. In addition, the TGF- $\beta$ R 1 mRNA expression by RT-qPCR in HTR-8/Svneo cells transfected with si-TGF- $\beta$ R1 was detected (Fig. S2C). The results demonstrated that following TGF- $\beta$ R 1 knockdown, its expression level decreased significantly, which also indicated that its transfection was successful.

\section{Discussion}

Despite the pathological conditions and complications, such as proteinuria and maternal multi-organ dysfunction (including uteroplacental dysfunction, fetal growth restriction and neurological or hematological complications) $(7,8)$, accompanying PE-induced hypertension, the causes and mechanism of the development of $\mathrm{PE}$ is remains to be elucidated, reducing the chance of treating or preventing the disease. Only a few effective pharmacological agents have been developed for the treatment of PE and pregnancy termination or early delivery of the fetus and placenta seems to be the most definitive treatment to date (43-46). Thus, recent research has been directed towards identifying regulatory molecules, such as the non-coding RNAs, which have been shown to be involved in the development of PE and their molecular mechanism of regulation $(47,48)$, hoping that identified molecules can serve as biomarkers for non-invasive diagnosis and prognosis of the disease and a therapeutic target for its treatment.

circRNA has been linked to the development of PE and is currently being studied as a potential biomarker and therapeutic target for the treatment PE. For instance, ceRNA expression profiling in pre-eclampsia identifies hsa_circ_0036877 as a potential novel biomarker for early PE (49), circTRNC18 inhibits trophoblast cell migration and EMT by regulating miR-762/Grhl2 pathway in PE (24). hsa_circ_0026552 was reported in 2013 by Salzman (25), but its specific molecular mechanism in PE has not been studied. Therefore, the present study aimed at determining the role of hsa_circ_0026552 in the development of PE. At the beginning of the present study, it was found that the hsa_circ_0026552 was highly upregulated in PE samples. RT-qPCR analysis confirmed that the hsa_circ_0026552 was differentially expressed in PE clinical tissue samples, further implicating the role of hsa_circ_0026552 in the development of PE. In addition, results from the present study revealed that silencing hsa_circ_0026552 markedly improved the proliferative, migrative and invasive ability of the HTR-8/SVneo cell line, suggesting that hsa_circ_0026552 might be an effective therapeutic target for treating PE, for the first time to the best of the authors' knowledge.

circRNAs perform their regulatory role in one of the major ways that affect the regulatory function of miRNAs and they target gene expression through competitively binding to the target miRNA seed region in PE (50). For example, circTRNC18 inhibits trophoblast cell migration and EMT by regulating miR-762/Grhl2 pathway in PE (24). Downregulated circPAPPA suppresses the proliferation and invasion of trophoblast cells via the miR-384/STAT3 pathway (51). The present study predicted all the target miRNAs of hsa_circ_0026552 and selected suitable targets. It was confirmed that hsa_circ_0026552 competitively binds to miR-331-3p. Compared with other target genes of hsa_circ_0026552, the miR-331-3p has been previously reported as an oncogenic miRNA that inhibits the tumor-suppressive ability of the ST7L gene in pancreatic cancer (30). It has also been shown as a tumor suppressor that represses the invasive and migrative capacity of colorectal carcinoma cells through NRP2 post-transcriptional inhibition (30). Xuefang et al (52) investigated the miR-331-3p role in the progression of nasopharyngeal carcinoma and found that the miR-331-3p could significantly impede the proliferation of nasopharyngeal carcinoma cell and induce their apoptosis by regulating the F4B-PI3K-AKT signaling pathway. All the above studies have shown that miR-331-3p can have a significant effect on cell phenotype, including inhibiting cell proliferation, migration and invasion and promoting cell apoptosis. Therefore, in PE, it probably promotes the regeneration of trophoblast cells and inhibits the development of PE progression. Other genes may also serve a role in the development of PE, which will be analyzed in future research. The present study found that miR-331-3p is low-expressed in PE and when co-transfected with hsa_circ_0026552 in 


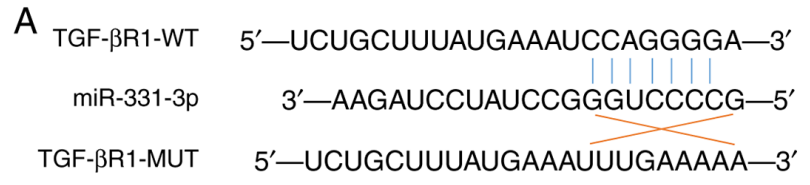

C
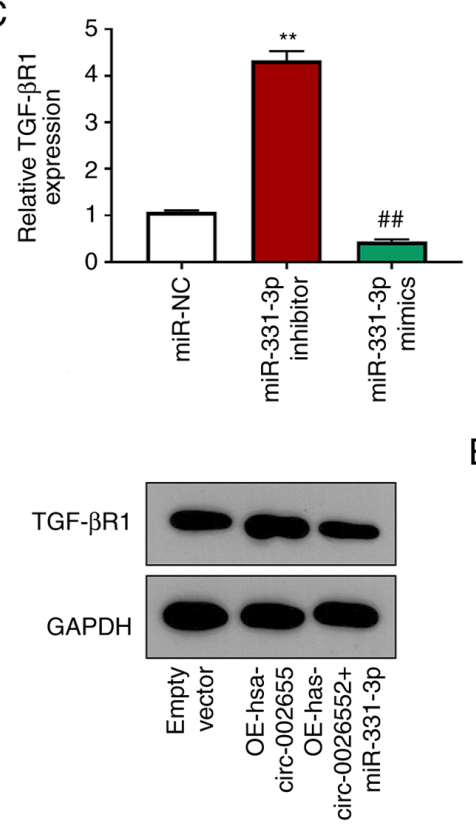

E
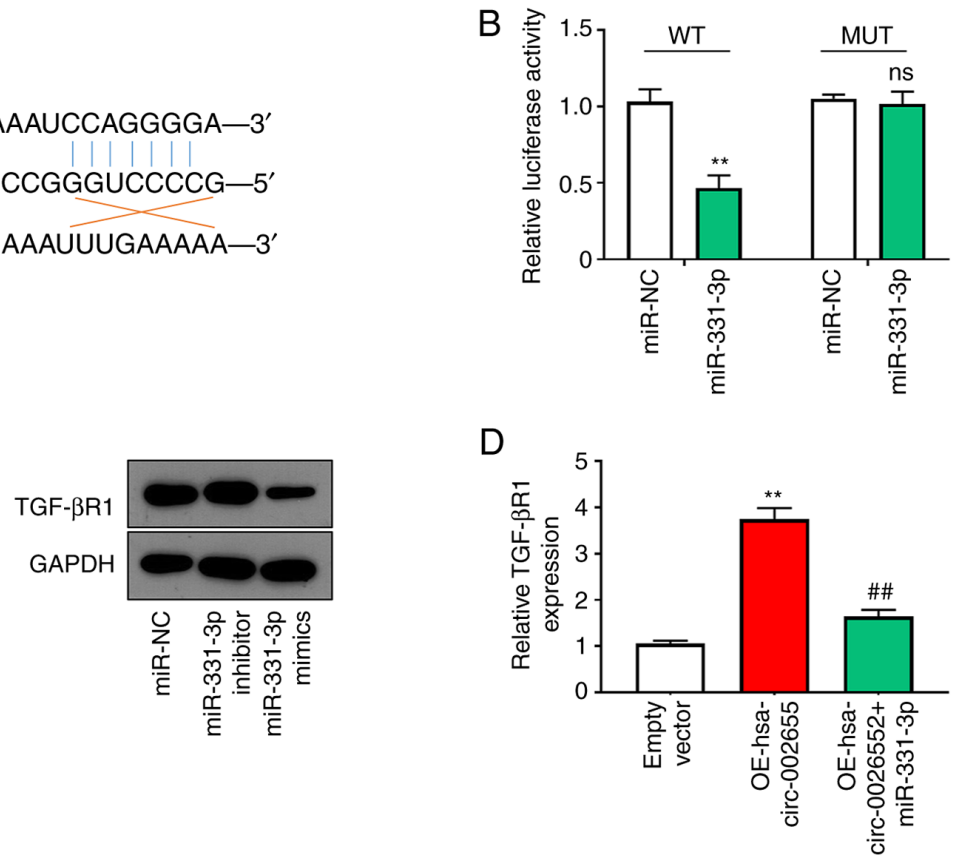

$\mathrm{F}$

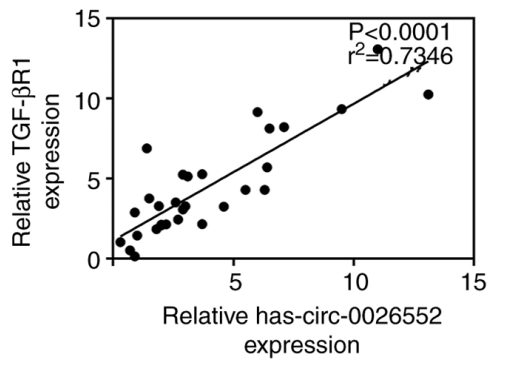

Figure 5. circRNA hsa_circ_0026552 regulates TGF- $\beta$ R1 expression in trophoblasts via sponging miR-331-3p. (A) Bioinformatics prediction of miR-331-3p target mRNA. (B) Dual-luciferase reporter assay showed the luciferase activity of HTR-8/Svneo cells co-transfected with the TGF- $\beta$ R1 WT plasmid and TGF- $\beta$ R1 MUT plasmid. (C) RT-qPCR and western blot analysis of TGF- $\beta$ R1 mRNA and protein expression following transfection. (D) RT-qPCR and western blot analysis of TGF- $\beta$ R1 expression in the HTR-8/Svneo cell after overexpressing hsa_circ_0026552 and miR-331-3p. (E) RT-qPCR analysis of TGF- $\beta$ R 1 differential expression in PE clinical tissue samples. (F) Correlation analysis of TGF- $\beta$ R1 and hsa_circ_0026552 expression in PE tissue samples. The experimental data are shown as mean \pm standard deviation of three independent experiments. ${ }^{* *} \mathrm{P}<0.01 ;{ }^{\# \#} \mathrm{P}<0.01$; ns, non-significant. circRNA, circular RNA; miR, microRNA; WT, wild type; MUT, mutant; RT-qPCR, reverse transcription quantitative PCR; PE, pre-eclampsia; NC, negative control; OE, overexpression.

HTR-8/SVneo cells, overexpression of miR-331-3p effectively reverses the effect of hsa_circ_0026552 on cell proliferation. This molecular regulation mechanism in PE is consistent with other studies $(43,52,53)$. In addition, the TGF- $\beta R 1$ gene was indicated as miR-331-3p target in the present study. The inhibition of this gene together with the TGF- $\beta /$ Smad signaling pathway has been shown to rescue the decline in the migrative and invasive property of trophoblast cells following DNMT3A knockdown (54). A previous study demonstrated that the aberrant expression of TGF- $\beta \mathrm{R} 1$ is strongly associated with a poor prognosis of patients diagnosed with pancreatic cancer and that TGF- $\beta$ R 1 might be a potential biomarker for the prognosis and treatment of pancreatic cancer (55). Notably, TGF- $\beta$ R1 has been studied in PE; Kim et al (33) indicated that the TGF- $\beta$ R1 polymorphism may be a genetic risk factor for PE and IUGR-complicated PE. The serum levels of TGF- $\beta$ R1 may contribute to the etiopathology of PE (38). However, the molecular mechanism in PE of TGF- $\beta$ R1 has not been deeply studied. Therefore, the present study found for the first time to the best of the authors' knowledge, that TGF- $\beta \mathrm{R} 1$ relative expression positively correlated with the expression of hsa_circ_0026552 and its upregulation might have inhibited the proliferative, migrative and invasive ability of trophoblasts.

The EMT is a key part of the embryonic development where epithelial cells lose their property and take on the migratory and invasive phenotype of mesenchymal cells (24). Studies have shown that a disruption in EMT-regulated migration and invasion contribute to PE (24). The present study noted that the overexpression of hsa_circ_0026552 significantly inhibited proliferation, migration and invasion of trophoblasts while miR-331-3p overexpression or TGF- $\beta$ R1 knockdown reversed this condition, suggesting that the overexpression of hsa_circ_0026552 could induce a dysfunctional EMT-regulated cell migration and invasion in trophoblasts which might result in the occurrence of PE. The overexpressed hsa_circ_0026552 inhibited the regulatory ability of miR-331-3p on TGF- $\beta$ R 1 and consequently upregulated TGF- $\beta$ R1 expression to promote PE. Taken together, the present study confirmed that the circRNA hsa_circ_0026552 was upregulated in $\mathrm{PE}$ and inhibited the proliferation, migration and invasion of trophoblast cells via the miR-331-3p/TGF- $\beta \mathrm{R} 1$ axis. The hsa_circ_0026552 might be involved in the development of $\mathrm{PE}$ and could be a possible therapeutic target for treating PE. 
A
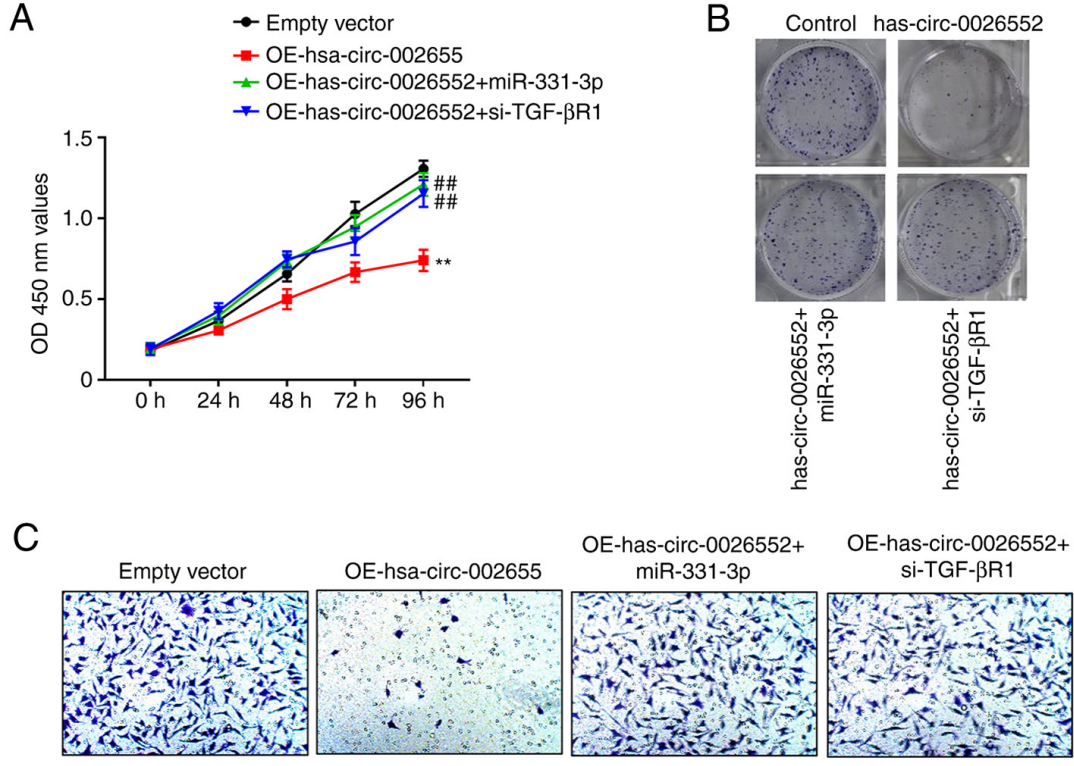

D

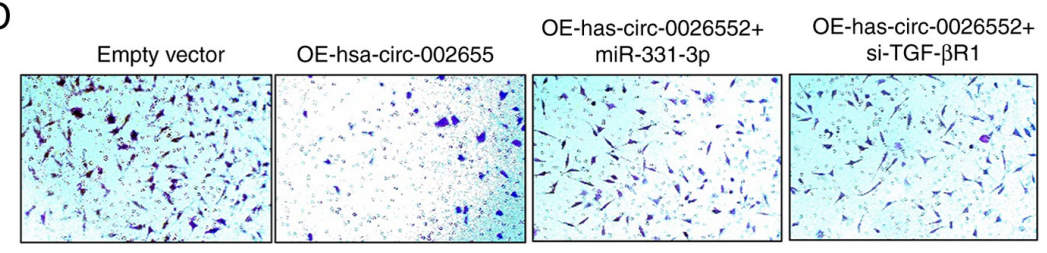

B
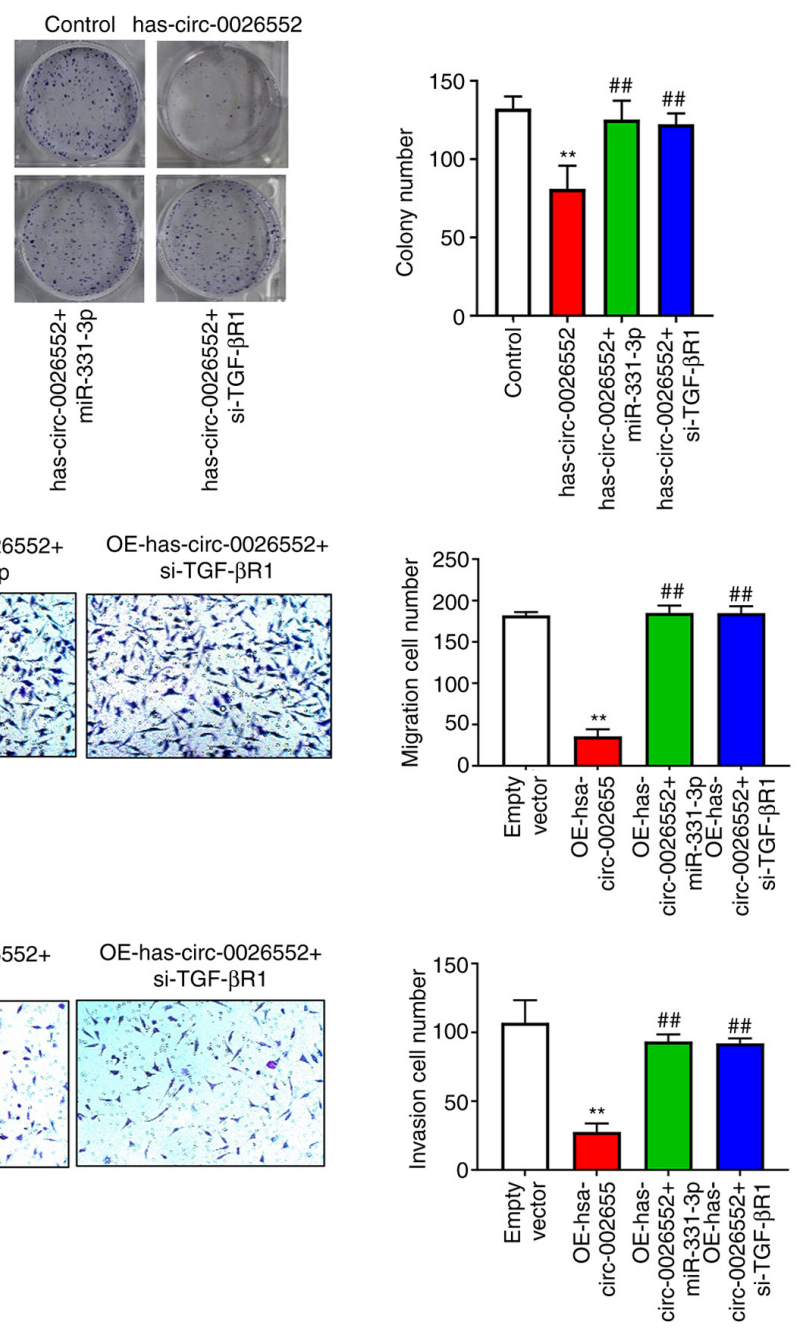

Figure 6. CircRNA hsa_circ_0026552 regulates the proliferation, migration and invasion of trophoblasts by targeting the miR-331-3p/ TGF- $\beta$ R1 axis. (A) CCK-8 and (B) colony formation assay analysis of the proliferation of HTR-8/Svneo cell lines following transfection. Transwell assay analysis of the (C) migration and (D) invasion of the HTR-8/Svneo cells following transfection. Magnification, $\mathrm{x} 200 .{ }^{* *} \mathrm{P}<0.01 ;{ }^{\# \#} \mathrm{P}<0.01$. circRNA, circular RNA; miR, microRNA; OE, overexpression.

The present study reported that hsa_circ_0026552 exhibited a significant upregulated expression in PE patients and promotes the migration, invasion and proliferation of HTR-8/SVneo cells by regulating the miR-331-3P/TGF- $\beta$ R 1 axis. The hsa_circ_0026552 could be a new therapeutic target for PE treatment. In addition, the proliferation, migration and invasion of trophoblast cells may be involved in PE development.

\section{Acknowledgements}

Not applicable.

\section{Funding}

No funding was received.

\section{Availability of data and materials}

All supporting data of this work, which are not available in public because of the ethical restrictions, are available from the corresponding author upon request.

\section{Authors' contributions}

LS designed and directed the experiments; LS and $\mathrm{XH}$ performed the experiments and data analysis. Both authors read and approved the final manuscript. LS and XH confirm the authenticity of all the raw data.

\section{Ethics approval and consent to participate}

The present study was authorized by the Ethics Committee of Yantaishan Hospital (approval no. KY-E-2020-1-10) and informed written consent was provided by all participants as per the guidelines of the Declaration of Helsinki.

\section{Patient consent for publication}

Not applicable.

\section{Competing interests}

The authors declare that they have no competing interests. 


\section{References}

1. Kurtz WS, Glueck CJ, Hutchins RK, Sisk RA and Wang P: Retinal artery and vein thrombotic occlusion during pregnancy: Markers for familial thrombophilia and adverse pregnancy outcomes. Clin Ophthalmol 10: 935-893, 2016.

2. Agius A, Sultana R, Camenzuli C, Calleja-Agius J and Balzan R: An update on the genetics of pre-eclampsia. Minerva Ginecol 70: 465-479, 2018.

3. Nakashima A, Cheng SB, Kusabiraki T, Motomura K, Aoki A, Ushijima A, Ono Y, Tsuda S, Shima T, Yoshino O, et al: Endoplasmic reticulum stress disrupts lysosomal homeostasis and induces blockade of autophagic flux in human trophoblasts Sci Rep 9: 11466, 2019.

4. Xu Y, Ge Z, Zhang E, Zuo Q, Huang S, Yang N, Wu D, Zhang Y, Chen Y, Xu H, et al: The lncRNA TUG1 modulates proliferation in trophoblast cells via epigenetic suppression of RND3. Cell Death Dis 8: e3104, 2017.

5. Mol BW, Roberts CT, Thangaratinam S, Magee LA, de Groot CJ and Hofmeyr GJ: Pre-eclampsia. Lancet 387: 999-1011, 2016

6. Poon LC, Shennan A, Hyett JA, Kapur A, Hadar E, Divakar H, McAuliffe F, da Silva Costa F, von Dadelszen P, McIntyre HD, et al: The international federation of gynecology and obstetrics (FIGO) initiative on pre-eclampsia: A pragmatic guide for first-trimester screening and prevention. Int J Gynaecol Obstet 145 (Suppl 1): S1-S33, 2019.

7. Phipps EA, Thadhani R, Benzing $\mathrm{T}$ and Karumanchi SA: Pre-eclampsia: Pathogenesis, novel diagnostics and therapies. Nat Rev Nephrol 15: 275-289, 2019.

8. Burton GJ, Redman CW, Roberts JM and Moffett A: Pre-eclampsia: Pathophysiology and clinical implications. BMJ 366: 12381, 2019.

9. Velicky P, Windsperger K, Petroczi K, Pils S, Reiter B, Weiss T, Vondra S, Ristl R, Dekan S, Fiala C, et al: Pregnancy-associated diamine oxidase originates from extravillous trophoblasts and is decreased in early-onset preeclampsia. Sci Rep 8: 6342, 2018.

10. Tong C, Feng X, Chen J, Qi X, Zhou L, Shi S, Kc K, Stanley JL, Baker PN and Zhang H: G protein-coupled receptor 30 regulates trophoblast invasion and its deficiency is associated with preeclampsia. J Hypertens 34: 710-718, 2016.

11. Wu L, Song WY, Xie Y, Hu LL, Hou XM, Wang R, Gao Y, Zhang JN, Zhang L, Li WW, et al: miR-181a-5p suppresses invasion and migration of HTR-8/SVneo cells by directly targeting IGF2BP2. Cell Death Dis 9: 16, 2018.

12. Wang G, Zhang Z, Chen C, Zhang Y and Zhang C: Dysfunction of WNT4/WNT5A in deciduas: Possible relevance to the pathogenesis of preeclampsia. J Hypertens 34: 719-727, 2016.

13. Chen LL and Yang L: Regulation of circRNA biogenesis. RNA Biol 12: 381-388, 2015.

14. Ebbesen KK, Kjems J and Hansen TB: Circular RNAs: Identification, biogenesis and function. Biochim Biophys Acta 1859: 163-168, 2016.

15. Hombach S and Kretz M: Non-coding RNAs: Classification, biology and functioning. Adv Exp Med Biol 937: 3-17, 2016.

16. Panda AC, Abdelmohsen K and Gorospe M: SASP regulation by noncoding RNA. Mech Ageing Dev 168: 37-43, 2017.

17. Qu S, Yang X, Li X, Wang J, Gao Y, Shang R, Sun W, Dou K and Li H: Circular RNA: A new star of noncoding RNAs. Cancer Lett 365: 141-148, 2015.

18. Zhang YG, Yang HL, Long Y and Li WL: Circular RNA in blood corpuscles combined with plasma protein factor for early prediction of pre-eclampsia. BJOG 123: 2113-2118, 2016.

19. Chen Q, Chen Z, Cao S, Guo B, Chen Y, Feng Z, Wang J, Guo G, Chen X and Huang X: Role of CircRNAs_100395 in proliferation and metastases of liver cancer. Med Sci Monit 25: 6181-6192, 2019.

20. Li R, Jiang J, Shi H, Qian H, Zhang X and Xu W: CircRNA: A rising star in gastric cancer. Cell Mol Life Sci 77: 1661-1680, 2020

21. Winship AL, Koga K, Menkhorst E, Van Sinderen M, Rainczuk K, Nagai M, Cuman C, Yap J, Zhang JG, Simmons D, et al Interleukin-11 alters placentation and causes preeclampsia features in mice. Proc Natl Acad Sci USA 112: 15928-15933, 2015.

22. Hansen YB, Myrhøj V, Jørgensen FS, Oxvig C and Sørensen S: First trimester PAPP-A2, PAPP-A and hCG $\beta$ in small-for-gestational-age pregnancies. Clin Chem Lab Med 54: 117-123, 2016.

23. Jiang M, Lash GE, Zhao X, Long Y, Guo $C$ and Yang $H$ : CircRNA-0004904, CircRNA-0001855, and PAPP-A: Potential novel biomarkers for the prediction of preeclampsia. Cell Physiol Biochem 46: 2576-2586, 2018.
24. Shen XY, Zheng LL, Huang J, Kong HF, Chang YJ, Wang F and Xin H: CircTRNC18 inhibits trophoblast cell migration and epithelial-mesenchymal transition by regulating miR-762/Grhl2 pathway in pre-eclampsia. RNA Biol 16: 1565-1573, 2019.

25. Salzman J, Chen RE, Olsen MN, Wang PL and Brown PO: Cell-type specific features of circular RNA expression. PLoS Genet 9: e1003777, 2013.

26. Wang Z and Liu Y: Predicting functional MicroRNA-mRNA interactions. Methods Mol Biol 1580: 117-126, 2017.

27. Wu HY, Wang XH, Liu K and Zhang JL: LncRNA MALAT1 regulates trophoblast cells migration and invasion via miR-206/IGF-1 axis. Cell Cycle 19: 39-52, 2020.

28. Gao Y, Guo X, Li Y, Sha W and She R: The decreased lncRNA ZEB2-AS1 in pre-eclampsia controls the trophoblastic cell line HTR-8/SVneo's invasive and migratory abilities via the miR-149/PGF axis. J Cell Biochem 120: 17677-17686, 2019.

29. Zhao M, Zhang M, Tao Z, Cao J, Wang L and Hu X: miR-331-3p suppresses cell proliferation in TNBC cells by downregulating NRP2. Technol Cancer Res Treat 19: 1533033820905824, 2020.

30. Zhang H, Wang R and Wang M: miR-331-3p suppresses cell invasion and migration in colorectal carcinoma by directly targeting NRP2. Oncol Lett 18: 6501-6508, 2019.

31. Jin W, Zhong N, Wang L, Yu J, Yin F and Zhang K: MiR-331-3p inhibition of the hepatocellular carcinoma (HCC) Bel-7402 cell line by down-regulation of E2F1. J Nanosci Nanotechnol 19: 5476-5482, 2019.

32. Pepper MS: Transforming growth factor-beta: Vasculogenesis, angiogenesis, and vessel wall integrity. Cytokine Growth Factor Rev 8: 21-43, 1997.

33. Kim SY, Lim JH, Park SY, Yang JH, Kim MY, Kim MH and Ryu HM. Transforming growth factor-beta 1 gene polymorphisms in Korean patients with pre-eclampsia. Am J Reprod Immunol 63: 291-8, 2010.

34. Brooks SA, Martin E, Smeester L, Grace MR, Boggess K and Fry RC: miRNAs as common regulators of the transforming growth factor (TGF)- $\beta$ pathway in the preeclamptic placenta and cadmium-treated trophoblasts: Links between the environment, the epigenome and preeclampsia. Food Chem Toxicol 98: 50-57, 2016.

35. Wang H, Zhang Q, Wang B, Wu W, Wei J, Li P and Huang R: miR-22 regulates $\mathrm{C} 2 \mathrm{C} 12$ myoblast proliferation and differentiation by targeting TGFBR1. Eur J Cell Biol 97: 257-268, 2018.

36. ElMonier AA, El-Boghdady NA, Abdelaziz MA and Shaheen AA: Association between endoglin/transforming growth factor beta receptors 1,2 gene polymorphisms and the level of soluble endoglin with preeclampsia in Egyptian women. Arch Biochem Biophys 662: 7-14, 2019.

37. Li X, Tan H, Chen M and Zhou S: Transforming growth factor beta 1 related gene polymorphisms in gestational hypertension and preeclampsia: A case-control candidate gene association study. Pregnancy Hypertens 12: 155-160, 2018.

38. Feizollahzadeh S, Taheripanah R, Khani M, Farokhi B and Amani D: Promoter region polymorphisms in the transforming growth factor beta-1 (TGF $\beta 1$ ) gene and serum TGF $\beta 1$ concentration in preeclamptic and control Iranian women. J Reprod Immunol 94: 216-221, 2012.

39. Shi Y, Guo Z, Fang N, Jiang W, Fan Y, He Y, Ma Z and Chen Y: hsa_circ_0006168 sponges miR-100 and regulates mTOR to promote the proliferation, migration and invasion of esophageal squamous cell carcinoma. Biomed Pharmacother 117: 109151, 2019.

40. Ye Y, Li SL and Wang SY: Construction and analysis of mRNA, miRNA, IncRNA, and TF regulatory networks reveal the key genes associated with prostate cancer. PLoS One 13: e0198055, 2018.

41. Wu Z, Huang W, Wang X, Wang T, Chen Y, Chen B, Liu R, Bai P and Xing J: Circular RNA CEP128 acts as a sponge of miR-145-5p in promoting the bladder cancer progression via regulating SOX11. Mol Med 24: 40, 2018

42. Song T, Xu A, Zhang Z, Gao F, Zhao L, Chen X, Gao J and Kong X: CircRNA hsa circRNA 101996 increases cervical cancer proliferation and invasion through activating TPX2 expression by restraining miR-8075. J Cell Physiol 234: 14296-14305, 2019.

43. Ou Y, Zhu L, Wei X, Bai S, Chen M, Chen H and Zhang J: Circular RNA circ_0111277 attenuates human trophoblast cell invasion and migration by regulating miR-494/HTRA1/Notch-1 signal pathway in pre-eclampsia. Cell Death Dis 11: 479, 2020.

44. Lu W, Ma YY, Shao QQ, Liang J, Qi TT, Huang Y and Wang QJ: ROS/p53/miR-335-5p/Sp1 axis modulates the migration and epithelial to mesenchymal transition of JEG-3 cells. Mol Med Rep 21: 1208-1216, 2020. 
45. Biró O, Nagy B and Rigó J Jr: Identifying miRNA regulatory mechanisms in preeclampsia by systems biology approaches. Hypertens Pregnancy 36: 90-99, 2017.

46. Li W, Yu N, Fan L, Chen SH and Wu JL: Circ_0063517 acts as ceRNA, targeting the miR-31-5p-ETBR axis to regulate angiogenesis of vascular endothelial cells in preeclampsia. Life Sci 244: 117306, 2020.

47. Bi W, Huang J, Nie C, Liu B, He G, Han J, Pang R, Ding Z, Xu J and Zhang J: CircRNA circRNA_102171 promotes papillary thyroid cancer progression through modulating CTNNBIP1-dependent activation of $\beta$-catenin pathway. J Exp Clin Cancer Res 37: 275, 2018.

48. Tang W, Fu K, Sun H, Rong D, Wang H and Cao H: CircRNA microarray profiling identifies a novel circulating biomarker for detection of gastric cancer. Mol Cancer 17: 137, 2018.

49. Hu X, Ao J, Li X, Zhang H, Wu J and Cheng W: Competing endogenous RNA expression profiling in pre-eclampsia identifies hsa_circ_0036877 as a potential novel blood biomarker for early pre-eclampsia. Clin Epigenetics 10: 48, 2018.

50. Chen X, Luo H, Li X, Tian X, Peng B, Liu S, Zhan T, Wan Y, Chen W, Li Y, et al: miR-331-3p functions as an oncogene by targeting ST7L in pancreatic cancer. Carcinogenesis 39: 1006-1015, 2018

51. Zhou W, Wang H, Yang J, Long W, Zhang B, Liu J and Yu B: Down-regulated circPAPPA suppresses the proliferation and invasion of trophoblast cells via the miR-384/STAT3 pathway. Biosci Rep: Sep 6,2019 (Epub ahead of print). doi: 10.1042/BSR20191965.
52. Xuefang Z, Ruinian Z, Liji J, Chun Z, Qiaolan Z, Jun J, Yuming C and Junrong H: miR-331-3p Inhibits Proliferation and Promotes Apoptosis of Nasopharyngeal Carcinoma Cells by Targeting elf4B-PI3K-AKT Pathway. Technol Cancer Res Treat. Jan-Dec 2020. doi: $10.1177 / 1533033819892251$.

53. Zhou B, Zhang X, Li T, Xie R, Zhou J, Luo Y and Yang C: CircZDHHC20 represses the proliferation, migration and invasion in trophoblast cells by miR-144/GRHL2 axis. Cancer Cell Int 20: 19, 2020.

54. Fan Y, Li T, Xu L and Kuang T: Comprehensive analysis of immunoinhibitors identifies LGALS9 and TGFBR1 as potential prognostic biomarkers for pancreatic cancer. Comput Math Methods Med 2020: 6138039, 2020.

55. Lamouille S, Xu J and Derynck R: Molecular mechanisms of epithelial-mesenchymal transition. Nat Rev Mol Cell Biol 15: 178-196, 2014.

This work is licensed under a Creative Commons Attribution-NonCommercial-NoDerivatives 4.0 International (CC BY-NC-ND 4.0) License. 